\title{
Reasoning about Threads Communicating via Locks
}

\author{
Vineet Kahlon, Franjo Ivančić and Aarti Gupta \\ NEC Labs America, \\ 4 Independence Way, \\ Princeton, NJ 08536, USA.
}

\begin{abstract}
We propose a new technique for the static analysis of concurrent programs comprised of multiple threads. In general, the problem is known to be undecidable even for programs with only two threads but where the threads communicate using CCS-style pairwise rendezvous [10]. However, in practice, a large fraction of concurrent programs can either be directly modeled as threads communicating solely using locks or can be reduced to such systems either by applying standard abstract interpretation techniques or by exploiting separation of control from data. For such a framework, we show that for the commonly occurring case of threads with nested access to locks, the problem is efficiently decidable. Our technique involves reducing the analysis of a concurrent program with multiple threads to individually analyzing augmented versions of the given threads. Thus not only yields decidability but also avoids construction of the state space of the concurrent program at hand and thus bypasses the state explosion problem making our technique scalable. We go on to show that for programs with threads that have non-nested access to locks, the static analysis problem for programs with even two threads becomes undecidable even for reachability, thus sharpening the result of [10]. As a case study, we consider the Daisy file system [1] which is a benchmark for analyzing the efficacy of different methodologies for debugging concurrent programs and show the existence of several bugs.
\end{abstract}

\section{Introduction}

Multi-threading is a standard way of enhancing performance by exploiting parallelism among the different components of a computer system. As a result the use of concurrent multi-threaded programs is becoming pervasive. Examples include operating systems, databases and embedded systems particularly for cellular phones. This necessitates the development of new methodologies to debug such systems especially since existing techniques for debugging sequential programs are inadequate in handling concurrent programs. The key reason for that is the presence of many possible interleavings among the local operations of individual threads giving rise to subtle unintended behaviors. This makes multi-threaded software behaviorally complex and hard to analyze thus requiring the use of formal methods for their validation.

One of the most widely used techniques in the validation of sequential programs is dataflow analysis [11] which can essentially be looked upon as a combination of abstract interpretation and model checking [12]. Here, abstract interpretation is used to get a finite representation of the control part of the program while recursion is modeled using a stack. Pushdown systems (PDSs) provide a natural framework to model such abstractly interpreted structures. A PDS has a finite control part corresponding to the 
valuation of the variables of the program and a stack which provides a means to model recursion. Dataflow analysis then exploits the fact that the model checking problem for PDSs is decidable for very expressive classes of properties - both linear and branching time (cf. $[2,15])$.

Following data-flow analysis for sequential programs, we model a multi-threaded program as a system comprised of multiple pushdown systems interacting with each other using a communication mechanism like a shared variable or a synchronization primitive ${ }^{1}$. While for a single PDS the model checking problem is efficiently decidable for very expressive logics, it was shown in [10] that even simple properties like reachability become undecidable even for systems with only two threads but where the threads communicate using CCS-style pairwise rendezvous.

However, in a large fraction of real-world concurrent software used, for example, in file systems, databases or device drivers, the key issue is to resolve conflicts between different threads competing for access to shared resources. Conflicts are typically resolved using locks which allow mutually exclusive access to a shared resource. Before a thread can have access to a shared resource it has to acquire the lock associated with that resource which is released after executing all the intended operations. For such software, the interaction between concurrently executing threads is very limited making them loosely coupled. For instance, in a standard file system the control flow in the implementation of the various file operations is usually independent of the data being written to or read from a file. Consequently such programs can either be directly modeled as systems comprised of PDSs communicating via locks or can be reduced to such systems either by applying standard abstract interpretation techniques or by exploiting separation of control and data. Therefore, in this paper, we consider the model checking problem for PDSs interacting using locks.

Absence of conflicts and deadlock freedom are among the most crucial properties that need to be checked for multi-threaded programs, particularly because checking for these is usually a pre-cursor for verifying more complex properties. Typical conflicts include, for example, data races where two or more threads try to access a shared memory location with at least one of the accesses being a write operation. This and most other commonly occurring conflicts (can be formulated to) occur pairwise among threads. With this in mind, given a concurrent program comprised of the $n$ threads $T_{1}, \ldots, T_{n}$, we consider correctness properties of the following forms:

- Liveness (Single-indexed Properties): E $h(i)$ and A $h(i)$, where $h(i)$ is an LTL $\backslash \mathrm{X}$ formula (built using F "eventually," U “until," G "always," but without X "nexttime") interpreted over the local control states of the PDS representing thread $T_{i}$, and $E$ (for some computation starting at the initial global configuration) and A (for all computations starting at the initial global configuration) are the usual path quantifiers.

- Safety (Double-indexed Properties): $\bigwedge_{i \neq j} \operatorname{EF}\left(a_{i} \wedge b_{j}\right)$, where $a_{i}$ and $b_{j}$ are local control states of PDSs $T_{i}$ and $T_{j}$, respectively.

- Deadlock Freedom.

\footnotetext{
${ }^{1}$ Henceforth we shall use the terms thread and PDS interchangeably.
} 
For single-indexed properties, we show that the model checking problem is efficiently decidable. Towards that end, given a correctness property E $h(i)$ over the local states of thread $T_{i}$, we show how to reduce reasoning in an exact, i.e., sound and complete, fashion about a system with $n$ threads to a system comprised of just the thread $T_{i}$. This reduces the problem of model checking a single-indexed LTL $\backslash X$ formula for a system with $n$ threads to model checking a single thread (PDS), which by [2] is known to be efficiently decidable.

The model checking problem for double-indexed properties is more interesting. As for single-indexed properties, we show that we can reduce the model checking problem for $\mathrm{E} h(i, j)$ for a system with $n$ threads to the system comprised of just the two threads $T_{i}$ and $T_{j}$. However, unlike the single index case, this still does not yield decidability of the associated model checking problem. We show that, in general, the problem of model checking remains undecidable even for pairwise reachability, viz., properties of the form $\operatorname{EF}\left(a_{i} \wedge b_{j}\right)$, where $a_{i}$ and $b_{j}$ are local control states of thread $T_{i}$ and $T_{j}$, even for programs with only two threads.

However, practical programming guidelines used by software developers often require that locks be used in a nested fashion, viz., each thread can only release the lock that it acquired last and that has not yet been released. Consequently most real-world concurrent programs have nested locks. In this case, we show that we can reduce reasoning about pairwise reachability of a given two-threaded program to individually model checking augmented versions of each of the threads which by [2] is efficiently decidable. The augmentation involves storing for each control location of a thread the history of locks that were acquired or released in order to get to that location. We show that storing this history information guarantees a sound and complete reduction. Furthermore, it avoids construction of the state space of the system at hand thereby bypassing the state explosion problem thus making our technique scalable to large programs. Thus we have given an efficient technique for reasoning about threads communicating via locks which can be combined synergistically with existing methodologies and can be easily incorporated into existing tools.

As a case study, we have applied our technique to check race conditions in the Daisy file system [1] and shown the existence of several bugs.

\section{System Model}

In this paper, we consider multi-threaded programs wherein threads communicate using locks. We model each thread using the trace flow graph framework (cf. [4]). Here each procedure of a thread is modeled as a flow graph, each node of which represents a control point of the procedure. The edges of the flow graph are annotated with statements that could either be assignments, calls to other procedures of the same thread or the acquire and release of locks when the thread needs to access shared resources. Recursion and mutual procedure calls are allowed. So that the flow graph of a program has a finite number of nodes, abstract interpretation techniques are often used in order to get a finite representation of the (potentially infinitely many) control states of the original thread. This typically introduces non-determinism which is explicitly allowed. Each thread can then be modeled as a system of flow graphs representing its procedures.

The resulting framework of finite state flow graphs with recursion can be naturally modeled as a pushdown system (PDS). A PDS has a finite control part corresponding 
to the valuation of the local variables of the procedure it represents and a stack which provides a means to model recursion.

Formally, a PDS is a five-tuple $\mathcal{P}=\left(P, A c t, \Gamma, c_{0}, \Delta\right)$, where $P$ is a finite set of control locations, Act is a finite set of actions, $\Gamma$ is a finite stack alphabet, and $\Delta \subseteq$ $(P \times \Gamma) \times A c t \times\left(P \times \Gamma^{*}\right)$ is a finite set of transition rules. If $\left((p, \gamma), a,\left(p^{\prime}, w\right)\right) \in \Delta$ then we write $\langle p, \gamma\rangle \stackrel{a}{\hookrightarrow}\left\langle p^{\prime}, w\right\rangle$. A configuration of $\mathcal{P}$ is a pair $\langle p, w\rangle$, where $p \in P$ denotes the control location and $w \in \Gamma^{*}$ the stack content. We call $c_{0}$ the initial configuration of $\mathcal{P}$. The set of all configurations of $\mathcal{P}$ is denoted by $\mathcal{C}$. For each action $a$, we define a relation $\stackrel{a}{\rightarrow} \subseteq \mathcal{C} \times \mathcal{C}$ as follows: if $\langle q, \gamma\rangle \stackrel{a}{\hookrightarrow}\left\langle q^{\prime}, w\right\rangle$, then $\langle q, \gamma v\rangle \stackrel{a}{\rightarrow}\left\langle q^{\prime}, w v\right\rangle$ for every $v \in \Gamma^{*}$.

We model multi-threaded programs using PDSs communicating using locks. For a concurrent program comprised of threads $T_{1}, \ldots, T_{n}$, a lock I is a globally shared variable taking on values from the set $\{1, \ldots, n, \perp\}$. The value of I can be modified by a thread using the operations acquire $(\mathrm{I})$ and release $(\mathrm{I})$. A thread can acquire a lock I only if its value is currently $\perp$, viz., none of the other threads currently has possession of it. Once I has been acquired by thread $T_{i}$, its value is set to $i$ and it remains so until $T_{i}$ releases it by executing release(l) thereby resetting its value to $\perp$. Locks are not pre-emptible, viz., a thread cannot be forced to give up any lock acquired by it.

Formally, we model a concurrent program with $n$ threads and $m$ locks $\mathrm{I}_{1}, \ldots, \mathrm{I}_{m}$ as a tuple of the form $\mathcal{C P}=\left(T_{1}, \ldots, T_{n}, L_{1}, \ldots, L_{m}\right)$, where $T_{1}, \ldots, T_{n}$ are pushdown systems (representing threads) with the same set Act of non-acquire and non-release actions, and for each $i, L_{i} \subseteq\{\perp, 1, \ldots, n\}$ is the possible set of values that lock $\mathrm{I}_{i}$ can be assigned. A global configuration of $\mathcal{C P}$ is a tuple $c=\left(t_{1}, \ldots, t_{n}, l_{1}, \ldots, l_{m}\right)$ where $t_{1}, \ldots, t_{n}$ are, respectively, the configurations of threads $T_{1}, \ldots, T_{n}$ and $l_{1}, \ldots, l_{m}$ the values of the locks. If no thread holds the lock in configuration $c$, then $l_{i}=\perp$, else $l_{i}$ is the index of the thread currently holding the lock. The initial global configuration of $\mathcal{C P}$ is $(c_{1}, \ldots, c_{n}, \underbrace{\perp, \ldots, \perp}_{m})$, where $c_{i}$ is the initial configuration of thread $T_{i}$. Thus all locks are free to start with. We extend the relation $\stackrel{a}{\longrightarrow}$ to pairs of global configurations as follows: Let $c=\left(c_{1}, \ldots, c_{n}, l_{1}, \ldots, l_{m}\right)$ and $c^{\prime}=\left(c_{1}^{\prime}, \ldots, c_{n}^{\prime}, l_{1}^{\prime}, \ldots, l_{m}^{\prime}\right)$ be global configurations. Then

- $c \stackrel{a}{\longrightarrow} c^{\prime}$ if there exists $1 \leq i \leq n$ such that $c_{i} \stackrel{a}{\longrightarrow} c_{i}^{\prime}$, and for all $1 \leq j \leq n$ such that $i \neq j, c_{j}=c_{j}^{\prime}$, and for all $1 \leq k \leq m, l_{k}=l_{k}^{\prime}$.

$-c \stackrel{\text { acquire }\left(\mathrm{I}_{i}\right)}{\longrightarrow} c^{\prime}$ if there exists $1 \leq j \leq n$ such that $c_{j} \stackrel{\text { acquire }\left(\mathrm{I}_{i}\right)}{\longrightarrow} c_{j}^{\prime}$, and $l_{i}=\perp$, and $l_{i}^{\prime}=j$, and for all $1 \leq k \leq n$ such that $k \neq j, c_{k}=c_{k}^{\prime}$, and for all $1 \leq p \leq m$ such that $p \neq i, l_{p}=l_{p}^{\prime}$.

$-c \stackrel{\text { release }\left(\mathrm{I}_{i}\right)}{\longrightarrow} c^{\prime}$ if there exists $1 \leq j \leq n$ such that $c_{j} \stackrel{\text { release }\left(\mathrm{I}_{i}\right)}{\longrightarrow} c_{j}^{\prime}$, and $l_{i}=j$, and $l_{i}^{\prime}=\perp$, and for all $1 \leq k \leq n$ such that $k \neq j, c_{k}=c_{k}^{\prime}$, and for all $1 \leq p \leq m$ such that $p \neq i, l_{p}=l_{p}^{\prime}$.

A sequence $x=x_{1}, x_{2}, \ldots$ of global configurations of $\mathcal{C P}$ is a computation if $x_{1}$ is the initial global configuration of $\mathcal{C P}$ and for each $i, x_{i} \stackrel{a}{\rightarrow} x_{i+1}$, where either $a \in$ Act or for some $1 \leq j \leq m, a=$ release $\left(\mathrm{I}_{j}\right)$ or $a=\operatorname{acquire}\left(\mathrm{I}_{j}\right)$. Given a thread $T_{i}$ and a 


\section{Safety Properties}

Even though proposition 2 allows us to reduce reasoning about double-indexed LTL $\backslash X$ properties from a system with $n$ threads to one with 2 threads, it still does not yield decidability. This is because although the model checking of LTL $\backslash X$ is decidable for a single pushdown system, it becomes undecidable even for simple reachability and even for systems with only two PDSs communicating via pairwise rendezvous [10]. The proof of undecidability rests on the fact that synchronization using pairwise rendezvous couples the two PDSs tightly enough to allow construction of a system that accepts the intersection of the two given context free languages (CFLs) the non-emptiness of which is undecidable.

We show that if we allow PDSs with non-nested lock access then the coupling, though seemingly weaker than pairwise rendezvous, is still strong enough to build a system accepting the intersection of the CFLs corresponding to the given PDSs thus yielding undecidability for even pairwise reachability. This is discouraging from a practical standpoint. However we exploit the observation that in most real-world concurrent programs locks are accessed by threads in a nested fashion. In that case, we can reduce the model checking of LTL $\backslash X$ properties in a sound and complete fashion for a concurrent program comprised of two threads to individually model checking augmented versions of the thread for LTL $\backslash X$ properties, which by [2] is efficiently decidable. Then combining this with the reduction result of the previous section, we get that the model checking problem of doubly-indexed LTL $\backslash \mathrm{X}$ formulas is efficiently decidable for concurrent programs with nested locks.

\subsection{Decidability of Pairwise Reachability for Nested Programs}

We motivate our technique with the help of a simple concurrent program $\mathcal{C P}$ shown below comprised of thread one with procedures thread_one and acq_rel_c, and thread two with procedures thread_two and acq_rel_b.

Suppose that we are interested in deciding whether $\operatorname{EF}(c 4 \wedge g 4)$ holds. The key idea is to reduce this to checking EF $c 4$ and $\mathrm{EF} g 4$ individually on (modifications of) the two threads. Then given computations $x$ and $y$ leading to $c 4$ and $g 4$, respectively, we merge them to construct a computation $z$ of $\mathcal{C P}$ leading to a global configuration with threads one and two in local control states $c 4$ and $g 4$, respectively. Consider, for example, the computations $x: \mathrm{c} 1, \mathrm{c} 2, \mathrm{~d} 1, \mathrm{~d} 2, \mathrm{c} 3, \mathrm{c} 4$ and $y: \mathrm{g} 1, \mathrm{~g} 2, \mathrm{~g} 3, \mathrm{~h} 1, \mathrm{~h} 2, \mathrm{~g} 4$ of threads one and two, respectively. Note that at control location $g 4$, thread 2 holds locks $\mathrm{c}$ and d. Also, along computation $y$ once thread two acquires lock $\mathrm{c}$ at control location $\mathrm{g} 1$, it does not release it and so we have to make sure that before we let it execute $g 1$ along $z$, all operations that acquire and release lock $\mathrm{c}$ along $x$ should already have been executed. Thus in our case $\mathrm{g} 1$ must be scheduled to fire only after $\mathrm{d} 2$ (and hence $\mathrm{c} 1$, $\mathrm{c} 2$ and $\mathrm{d} 1$ ) have already been executed. Similarly, operation c 3 of thread one must be executed after $\mathrm{h} 2$ has already been fired along $z$. Thus one possible computation $z$ of $\mathcal{C P}$ with the desired properties is $z: \mathrm{c} 1, \mathrm{c} 2, \mathrm{~d} 1, \mathrm{~d} 2, \mathrm{~g} 1, \mathrm{~g} 2, \mathrm{~g} 3, \mathrm{~h} 1$, $\mathrm{h} 2, \mathrm{~g} 4, \mathrm{c} 3, \mathrm{c} 4$.

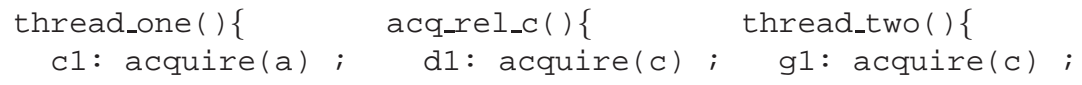




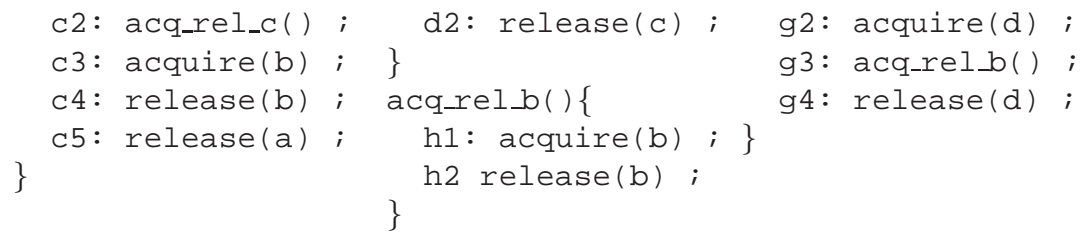

Note that if we replace the function call at control location $\mathrm{g} 3$ of thread two by acq_rel_a () which first acquires and then releases lock a, then there is no way to reconcile the two local computations $x$ and $y$ to get a global computation leading to a configuration with threads one and two, respectively, at control locations $c 4$ and $g 4$, even though they are reachable in their respective individual threads. This is because in this case $\mathrm{h} 2$ (and hence $\mathrm{g} 1, \mathrm{~g} 2, \mathrm{~g} 3$ and $\mathrm{h} 1$ ) should be executed before $\mathrm{c} 1$ (and hence $c 2, d 1, d 2, c 3$ and $c 4)$. Again, as before, $g 1$ can be fired only after $d 2$ (and hence $\mathrm{c} 1, \mathrm{c} 2$ and $\mathrm{d} 1$ ). From the above observations we get that $\mathrm{g} 1$ must be fired after $\mathrm{h} 2$ along $z$. But that violates the local ordering of the transitions fired along $y$ wherein $\mathrm{g} 1$ was fired before $\mathrm{h} 2$. This proves the claim made above.

In general when testing for reachability of control states $c$ and $c^{\prime}$ of two different threads it suffices to test whether there exist paths $x$ and $y$ in the individual threads leading to states $c$ and $c^{\prime}$ holding lock sets $L$ and $L^{\prime}$ which can be acquired in a compatible fashion. Compatibility ensures that we do not get a scenario as above where there exist locks $a \in L$ and $a^{\prime} \in L^{\prime}$ such that a transition acquiring $a^{\prime}$ was fired after acquiring $a$ for the last time along $x$ and a transition acquiring $a$ was fired after acquiring $a^{\prime}$ for the last time along $y$, else we can't reconcile $x$ and $y$. The above discussion is formalized below in theorem 5 the proof of which is given in the appendix. Before proceeding further, we need the following definition.

Definition 4 (Acquisition History) Let $x$ be a global computation of a concurrent program $\mathcal{C P}$ leading to global configuration $c$. Then for thread $T_{i}$ and lock $l_{j}$ of $\mathcal{C P}$ such that $j \in \operatorname{Lock-Set}\left(T_{i}, c\right)$, we define $A H\left(T_{i}, l_{j}, x\right)$ to be the set of indices of locks that were acquired (and possibly released) by $T_{i}$ after the last acquisition of $l_{j}$ by $T_{i}$ along $x$.

Theorem 5 (Decomposition Result) Let $\mathcal{C P}$ be a concurrent program comprised of the two threads $T_{1}$ and $T_{2}$ with nested locks. Then for control states $a_{1}$ and $b_{2}$ of $T_{1}$ and $T_{2}$, respectively, $\mathcal{C P} \models \operatorname{EF}\left(a_{1} \wedge b_{2}\right)$ iff there are computations $x$ and $y$ of the individual threads $T_{1}$ and $T_{2}$, respectively, leading to configurations $s$ with $T_{1}$ in control state $a_{1}$ and $t$ with $T_{2}$ in control state $b_{2}$ such that

- Lock-Set $\left(T_{1}, s\right) \cap \operatorname{Lock-Set}\left(T_{2}, t\right)=\emptyset$.

- there do not exist locks $l \in \operatorname{Lock-Set}\left(T_{1}, s\right)$ and $l^{\prime} \in \operatorname{Lock-Set}\left(T_{2}, t\right)$ with $l^{\prime} \in$ $A H\left(T_{1}, l, x\right)$ and $l \in A H\left(T_{2}, l^{\prime}, y\right)$.

To make use of the above result we augment the given threads to keep track of the acquisition histories. Given a thread $\mathcal{P}=\left(P, A c t, \Gamma, c_{0}, \Delta\right)$ of concurrent program $\mathcal{C P}$ having the set of locks $L$ of cardinality $m$, we construct the augmented thread $\mathcal{P}_{A}=\left(P_{A}, A c t, \Gamma, d_{0}, \Delta_{A}\right)$, where $P_{A}=P \times 2^{L} \times\left(2^{L}\right)^{m}$ and $\Delta_{A} \subseteq\left(P_{A} \times \Gamma\right) \times$ $\left(P_{A} \times \Gamma^{*}\right)$. The augmented PDA is used to track the set of locks and acquisition history of thread $T$ along local computations of $T$. Let $x$ be a computation of $\mathcal{C P}$ leading to 
global configuration $s$. Each control location of the augmented PDA is of the form $\left(a, L o c k s, A H_{1}, \ldots, A H_{m}\right)$, where $a$ denotes the current control state of $T$ in $s$, Locks the set of locks currently held by $T$ and for $1 \leq j \leq m$, if $l_{j} \in \operatorname{Locks}$, then $A H_{j}$ is the set $A H\left(T, l_{j}, x\right)$ else it is the empty set. The initial configuration $d_{0}$ is the $(m+2)$-tuple $\left(c_{0}, \emptyset, \emptyset, \ldots, \emptyset\right)$. The transition relation $\Delta_{A}$ is defined as follows:

$$
\left\langle q, \text { Locks }, A H_{1}, \ldots, A H_{m}, \gamma\right\rangle \stackrel{a}{\hookrightarrow}\left\langle q^{\prime}, L o c k s^{\prime}, A H_{1}^{\prime}, \ldots, A H_{m}^{\prime}, w\right\rangle \in \Delta_{A} \text { iff }
$$

- $a$ is not a lock operation, $\langle q, \gamma\rangle \stackrel{a}{\hookrightarrow}\left\langle q^{\prime}, w\right\rangle \in \Delta$, Locks $=$ Locks $s^{\prime}$ and for $1 \leq j \leq$ $m, A H_{j}=A H_{j}^{\prime}$.

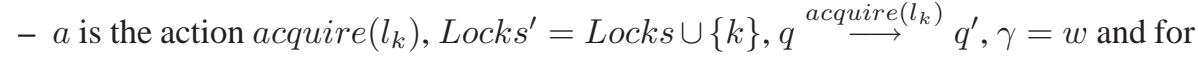
$1 \leq p \leq m$, if $p \in$ Locks then $A H_{p}^{\prime}=A H_{p} \cup\{k\}$ and $A H_{p}^{\prime}=A H_{p}$ otherwise.

- $a$ is the action release $\left(l_{k}\right)$, Lock $k^{\prime}=\operatorname{Locks} \backslash\{k\}, q \stackrel{\text { release }\left(l_{k}\right)}{\longrightarrow} q^{\prime}, \gamma=w, A H_{k}^{\prime}=\emptyset$ and for $1 \leq p \leq m$ such that $p \neq k, A H_{p}^{\prime}=A H_{p}$.

Proposition 6 Given a concurrent program $\mathcal{C P}$ comprised of threads $T$ and $T^{\prime}$, the model checking problem for pairwise reachability, viz., formulas of the form $\operatorname{EF}\left(a_{1} \wedge b_{2}\right)$ is decidable.

Implementation Issues. Given a concurrent program, to implement our technique we introduce for each lock 1 two extra global variables defined as follows:

1. possession_l: to track whether 1 is currently in the possession of a thread

2. history_l: to track the acquisition history of 1 .

To begin with, possession_l is initialized to false and history_l to the emptyset. Then each statement of the form acquire $(1 \mathrm{k})$ in the original code is replaced by the following statements:

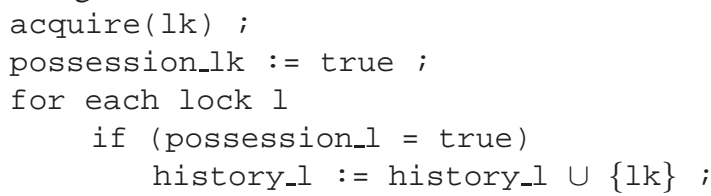

Similarly, each statement of the form release $(1 \mathrm{k})$ is replaced with the following sequence of statements:

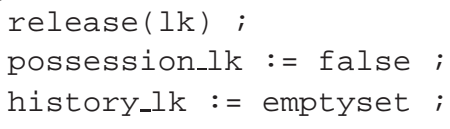

Optimizations. The above naive implementation keeps the acquisition history for each lock of the concurrent program and tests for all possible disjoint pairs $L$ and $L^{\prime}$ of lock sets and all possible compatible acquisition histories at two given error control locations $a_{i}$ and $b_{j}$, say. In the worst case this is exponential in the number of locks. However by exploiting program analysis techniques one can severely cut down on the number of such lock sets and acquisition histories that need to be tested for each control location of the given program as discussed below.

Combining Lock Analysis with Program Analysis: Using static analysis on the control flow graph of a given thread we can get a conservative estimate of the set of locks that could possibly have been acquired by a thread with its program counter at a given 
control location $c$. This gives us a superset $L_{c}$ of the set of locks that could possibly have been acquired at control location $c$ and also the possible acquisition histories. Thus in performing the reachability analysis, $\operatorname{EF}\left(a_{i} \wedge b_{j}\right)$, we only need to consider sets $L$ and $L^{\prime}$ of locks such that $L \cap L^{\prime}=\emptyset, L \subseteq L_{a_{i}}$ and $L^{\prime} \subseteq L_{b_{j}}$. This can exponentially cut down on the lock sets and acquisition histories that need be explored as, in practice, the nesting depth of locks is usually one and so the cardinality of $L_{c}$ will usually be one.

Combining Lock Analysis with Program Slicing By theorem 5, for a control location $c$ of thread $T$ we only need to track histories of only those locks that are in possession of $T$ at $c$ instead of every lock as was done in the naive implementation. Furthermore for a lock $l$ in possession of $T$ at $c$ we can ignore all lock operations performed by $T$ before $l$ was acquired for the last time by $T$ before reaching $c$ as these don't affect the acquisition history of $l$. Such statements can thus be deleted using program slicing techniques.

\section{Deadlockability}

In our framework, since synchronization among threads is carried out using locks, the only way a deadlock can occur is if there is a reachable global state $s$ with a dependency cycle of the form $T_{i_{1}} \rightarrow T_{i_{2}} \rightarrow \ldots \rightarrow T_{i_{p}} \rightarrow T_{i_{1}}$, where $T_{i_{k-1}} \rightarrow T_{i_{k}}$ if the program counter of $T_{i_{k-1}}$ is currently at an acquire operation for a lock that is currently held by $T_{i_{k}}$. Thus to decide whether any thread in the given program $\mathcal{C P}$ is deadlockable, for each thread $T_{i}$ of $\mathcal{C P}$ we first construct the set of reachable configurations of the corresponding augmented thread $\left(T_{i}\right)_{A}$ (defined above) in which the control location corresponds to an acquire operation. Denote the set of such configurations by $A c q_{i}$. We then construct a directed graph $D_{\mathcal{C P}}$ whose nodes are elements of the set $\bigcup_{i} A c q_{i}$ and there is a directed edge from configuration $\mathbf{a}=\left(a, L, A H_{1}, \ldots, A H_{m}\right)$ to $\mathbf{a}^{\prime}=$ $\left(a^{\prime}, L^{\prime}, A H_{1}^{\prime}, \ldots, A H_{m}^{\prime}\right)$ iff there exist $i \neq i^{\prime}$ such that (i) $\mathbf{a} \in A c q_{i}, \mathbf{a}^{\prime} \in A c q_{i^{\prime}}$, and (ii) $a$ and $a^{\prime}$ both correspond to acquire operations, say, acquire $(l)$ and acquire $\left(l^{\prime}\right)$, respectively, and (iii) thread $T_{i^{\prime}}$ currently holds lock $l$ required by thread $T_{i}$, viz., $l \in L^{\prime}$. Then the given current program is deadlockable iff there exists a cycle $\mathbf{c}_{1} \rightarrow \ldots \rightarrow$ $\mathbf{c}_{p} \rightarrow \mathbf{c}_{1}$ in $D_{\mathcal{C P}}$ such that every pair of configurations $\mathbf{c}_{j}=\left(c_{j}, L_{j}, A H_{j 1}, \ldots, A H_{j m}\right)$ and $\mathbf{c}_{j^{\prime}}=\left(c_{j^{\prime}}, L_{j^{\prime}}, A H_{j^{\prime} 1}, \ldots, A H_{j^{\prime} m}\right)$ is consistent, viz., $L_{j} \cap L_{j^{\prime}}=\emptyset$ and there do not exists locks $l_{r} \in L_{j}$ and $l_{r^{\prime}} \in L_{j^{\prime}}$ such that $l_{r^{\prime}} \in A H_{j r}$ and $l_{r} \in A H_{j^{\prime} r^{\prime}}$. By theorem 5, consistency ensures that the global state encompassing the cycle is a reachable state of $\mathcal{C P}$. Note that again we have bypassed the state explosion problem by not constructing the state space of the system at hand. Furthermore using the optimizations discussed in the previous section, we can severely cut down on the possible lock sets and acquisition histories that we need to track for each acquire location in each thread. This ensures that the size of $D_{\mathcal{C P}}$ remains tractable.

\section{Undecidability for Programs with Non-nested Locks}

In this section, we show that for concurrent programs comprised of two threads $T_{1}$ and $T_{2}$ communicating via locks (not necessarily nested), the model checking problem for pairwise reachability, viz., properties of the form $\operatorname{EF}\left(a_{1} \wedge b_{2}\right)$, where $a_{1}$ and $b_{2}$ are control states of $T_{1}$ and $T_{2}$, respectively, is undecidable. 
Given a concurrent program $\mathcal{C P}$ comprised of two threads $T_{1}$ and $T_{2}$ communicating via pairwise rendezvous, we construct a new concurrent program $\mathcal{C} \mathcal{P}^{\prime}$ comprised of threads $T_{1}$ and $T_{2}$ by (weakly) simulating pairwise rendezvous using non-nested locks such that the set of control states of $\mathrm{T}_{1}$ and $\mathrm{T}_{2}$ are supersets of the sets of control states of $T_{1}$ and $T_{2}$, respectively, and for control states $a_{1}$ and $b_{2}$ of $T_{1}$ and $T_{2}$, respectively, $\mathcal{C P} \models \operatorname{EF}\left(a_{1} \wedge b_{2}\right)$ iff $\mathcal{C} \mathcal{P}^{\prime} \models \operatorname{EF}\left(a_{1} \wedge b_{2}\right)$. This reduces the decision problem for pairwise reachability for threads communicating via pairwise rendezvous to threads communicating via locks. But since pairwise reachability for threads communicating via pairwise rendezvous is undecidable, our result follows.

Simulating Pairwise Rendezvous using Locks We now present the key idea behind the simulation leaving the details for the appendix. We show how to simulate a given pair $a \stackrel{m !}{\longrightarrow} b$ and $c \stackrel{m ?}{\longrightarrow} d$ of send and receive pairwise rendezvous transitions, respectively. Recall that for this rendezvous to be executed, both the send and receive transitions must be simultaneously enabled, else neither transition can fire. Corresponding to the labels $m$ ! and $m$ ?, we first introduce the new locks $l_{m !}, l_{m}$ ? and $l_{m}$.

Consider now the send transition $\operatorname{tr}: a \stackrel{m !}{\longrightarrow} b$ of thread $T_{1}$, say. Our construction (see details in appendix) ensures that before $\mathrm{T}_{1}$ starts mimicking $t r$ in local state $a$ it already has possession of lock $l_{m}$ ? . Then $\mathrm{T}_{1}$ simulates $T_{1}$ via the following sequence of transitions: $a \stackrel{\text { acquire }\left(l_{m}\right)}{\longrightarrow} a_{1} \stackrel{\text { release }\left(l_{m}\right)}{\longrightarrow} a_{2} \stackrel{\text { acquire }\left(l_{m !}\right)}{\longrightarrow} a_{3} \stackrel{\text { release }\left(l_{m}\right)}{\longrightarrow} b \stackrel{\text { acquire }\left(l_{m} \text { ? }\right)}{\longrightarrow}$ $b_{1} \stackrel{\text { release }\left(l_{m !}\right)}{\longrightarrow} b_{2}$. Similarly we assume that $\mathrm{T}_{2}$ has possession of $l_{m}$ ! before it starts mimicking $t r^{\prime}: c \stackrel{m ?}{\longrightarrow} d$. Then $\mathrm{T}_{2}$ simulates $t r^{\prime}$ by firing the following sequence of transitions: $c \stackrel{\text { acquire }\left(l_{m}\right)}{\longrightarrow} c_{1} \stackrel{\text { release }\left(l_{m !}\right)}{\longrightarrow} c_{2} \stackrel{\text { acquire }\left(l_{m}\right)}{\longrightarrow} c_{3} \stackrel{\text { release }\left(l_{m} ?\right)}{\longrightarrow} d \stackrel{\text { acquire }\left(l_{m !}\right)}{\longrightarrow}$ $d_{1} \stackrel{\text { release }\left(l_{m}\right)}{\longrightarrow} d_{2}$.

The reason for letting thread $\mathrm{T}_{1}$ acquire $l_{m}$ ? at the outset is to prevent thread $\mathrm{T}_{2}$ from firing transition $c \stackrel{m ?}{\longrightarrow} d$ without synchronizing with $t r: a \stackrel{m !}{\longrightarrow} d$. To initiate execution of the pairwise rendezvous involving $t r$, thread $\mathrm{T}_{1}$ releases lock $l_{m}$ ? and only when lock $l_{m}$ ? is released can $\mathrm{T}_{2}$ pick it up in order to execute the matching receive transition labeled with $m$ ?. But before $\mathrm{T}_{1}$ releases $l_{m}$ ? it acquires $l_{m}$. Note that this trick involving chaining wherein before releasing a lock a thread is forced to pick up another lock gives us the ability to introduce a relative ordering on the firing of local transitions of $T_{1}$ and $T_{2}$ which in turn allows us to simulate (in a weak sense) the firing of the pairwise rendezvous comprised of $t r$ and $t r^{\prime}$. It can be seen that due to chaining, the local transitions in the two sequences defined above can only be interleaved in the following order: $a \stackrel{\text { acquire }\left(l_{m}\right)}{\longrightarrow} a_{1}, a_{1} \stackrel{\text { release }\left(l_{m} \text { ? }\right)}{\longrightarrow} a_{2}, \stackrel{\text { acquire }\left(l_{m} \text { ? }\right)}{\longrightarrow} c_{1}, c_{1} \stackrel{\text { release }\left(l_{m !}\right)}{\longrightarrow} c_{2}$, $a_{2} \stackrel{\text { acquire }\left(l_{m !}\right)}{\longrightarrow} a_{3} a_{3} \stackrel{\text { release }\left(l_{m}\right)}{\longrightarrow} b, c_{2} \stackrel{\text { acquire }\left(l_{m}\right)}{\longrightarrow} c_{3}, c_{3} \stackrel{\text { release }\left(l_{m} ?\right)}{\longrightarrow} d, b \stackrel{\text { acquire }\left(l_{m}\right)}{\longrightarrow} b_{1}$, $b_{1} \stackrel{\text { release }\left(l_{m !}\right)}{\longrightarrow} b_{2}, d \stackrel{\text { acquire }\left(l_{m !}\right)}{\longrightarrow} d_{1}, d_{1} \stackrel{\text { release }\left(l_{m}\right)}{\longrightarrow} d_{2}$.

It is important to note that the use of overlapping locks is essential in implementing chaining thereby forcing a pre-determined order of firing of the local transitions which cannot be accomplished using nested locks alone. Although the above construction captures the key idea behind the simulation, there are other technical intricacies that require augmentation of the above construction and are discussed in the appendix. 
Since the model checking problem for pairwise reachability is known to be undecidable for threads communicating using pairwise rendezvous [10] and since we can, by the above result, simulate pairwise rendezvous using locks in a way so as to preserve pairwise reachability, we have the following undecidability result.

Theorem 8 The model checking problem for pairwise reachability is undecidable for concurrent programs comprised of two threads communicating using locks.

\section{The Daisy Case Study}

We have used our technique to find bugs in the Daisy file system which is a benchmark for analyzing the efficacy of different methodologies for verifying concurrent programs [1]. Daisy is a 1KLOC Java implementation of a toy file system where each file is allocated a unique inode that stores the file parameters and a unique block which stores data. An interesting feature of Daisy is that it has fine grained locking in that access to each file, inode or block is guarded by a dedicated lock. Moreover, the acquire and release of each of these locks is guarded by a 'token' lock. Thus control locations in the program might possibly have multiple open locks and furthermore the acquire and release of a given lock can occur in different procedures.

We are currently incorporating our lock analysis technique into F-Soft [8] which is a framework for model checking sequential software. So far we have implemented the decision procedure for pairwise reachability and used it to detect race conditions in the Daisy file system. Towards that end we check that for all $n$, any $n$-threaded Daisy program does not have a given race condition. Since a race condition can be expressed as pairwise reachability, using proposition 1, we see that it suffices to check a 2-thread instance which is done using our procedure involving acquisition histories. We have shown the existence of the following race conditions also noted by other researchers (cf. [1]) :

1. Daisy maintains an allocation area where for each block in the file system a bit is assigned 0 or 1 accordingly as the block has been allocated to a file or not. But each disk operation reads/writes an entire byte. Two threads accessing two different files might access two different blocks. However since bytes are not guarded by locks in order to set their allocation bits these two different threads may access the same byte in the allocation block containing the allocation bit for each of these locks thus setting up a race condition. For the data race described above, the statistics are as follows. The pre-processing phase which includes slicing, range analysis, using static analysis to find the possible set of open locks at the control state corresponding to the error label and then incorporating the acquisition history statements in the CFGs corresponding to the threads for only these locks took $77 \mathrm{secs}^{2}$ for both the threads. The two model checking runs took 5.3 and 21.67 secs and the error labels corresponding to the race condition were reached at depths 75 and 333, respectively in the two threads using SAT-based BMC in F-Soft.

2. In Daisy reading/writing a particular byte on the disk is broken down into two operations: a seek operation that mimics the positioning of the head and a read/write operation that transfers the actual data. Due to this separation between seeking and data transfer a race condition may occur. For example, read two disk locations, say $n$ and $m$,

\footnotetext{
${ }^{2}$ Machine Info: Intel Pentium4 3.20GHz CPU, 2MB RAM.
} 
we must make sure that $\operatorname{seek}(n)$ is followed by $\operatorname{read}(n)$ without $\operatorname{seek}(\mathrm{m})$ or $\operatorname{read}(\mathrm{m})$ scheduled in between. Here the pre-processing phase took about the same time as above. The model checking runs on the two threads took 15 and 35 secs.

We are currently in the process of implementing the other optimizations discussed in the paper, as well as the procedure for deadlock detection which will enable us to test our technique on yet larger real-world examples.

\section{Conclusion and Related Work}

In this paper, we have considered the static analysis of concurrent multi-threaded programs wherein the threads communicate via locks. We have shown that for single index LTL $\backslash X$ properties the problem is efficiently decidable. On the other hand, for double index LTL $\backslash X$ properties, the problem can be shown to be undecidable even for reachability and for systems with only two threads. However, for the practically important case where the locks are nested we get efficient decidability for pairwise reachability. We have implemented our technique in a prototype software verification platform and our preliminary results on the Daisy benchmark are encouraging.

There has been interesting prior work on extending data flow analysis to handle concurrent programs. In [3], the authors attempt to generalize the decision procedures given in [2] to handle pushdown systems communicating via CCS-style pairwise rendezvous. However since even reachability is undecidable for such a framework the procedures are not guaranteed to terminate in general but only for certain special cases, some of which the authors identify. However their practical utility is not clear.

A commonly used approach to cut down on the number of interleavings when reasoning about concurrent systems is to exploit syntactic independence among the local operations of different components of the system. In [9] this idea is exploited using the concept of transactions, wherein executing a transaction is equivalent to atomically executing a sequence of operations of each thread that do not involve communication with other threads and thus their execution does not interfere in the operation of other threads. The advantage of this technique is that it works well provided one can statically decide whether two given operations from two different threads are independent, a problem which is, in general, hard. The disadvantage is that although this technique can potentially cut down on the number of interleavings to be explored, it still does not completely address the core issue of state explosion. The use of partial order techniques $[6,5,13,14]$ also exploits syntactic independence of transitions to cut down on the number of interleavings to be explored and although extremely useful, suffers from the same drawback as above.

Another technique that has been adapted from concurrent protocol verification is the use of compositional reasoning wherein one tries to reduce reasoning about the correctness of a system comprised of many concurrently executing components to reasoning about each individual components. In [7] an assume-guarantee style reasoning is used to abstract out the environment of each thread in a purely automatic fashion for system where the threads are loosely coupled. A drawback is that the technique is not complete for reachability and thus is not guaranteed to find all errors. Furthermore, error trace recovery is hard because abstracting the environment causes a lot of information to be lost and thus it may not be possible to construct a concrete error trace purely from the environment assumptions. 
We, on the other hand, have identified a practically important case of threads communicating using locks and shown how to reason efficiently about a rich class of properties. We combat state explosion by reducing reasoning about indexed LTL $\backslash X$ properties and deadlockability to reasoning about individual threads. Our methods are exact, i.e., both sound and complete, and cater to automatic error trace recovery. A key advantage of our method is that by avoiding construction of the state space of the system at hand we bypass the state explosion problem, thus guaranteeing scalability of our approach. A potential drawback of our method is that it works only for threads that communicate using locks. However we believe that a very large fraction of concurrent software is loosely coupled and even where richer communication mechanisms are used, the interaction between the threads is not very subtle and can often, by using standard abstract interpretation techniques, be modeled as threads communicating solely using locks. Our technique can therefore be looked upon as a first line of attack in combating state explosion in the context of multi-threaded software providing an exact and efficient procedure for verifying an important class of multi-threaded software. Finally, our technique can easily be incorporated into existing tool flows.

\section{References}

1. Joint cav/issta special event on specification, verification, and testing of concurrent software. In http://research.microsoft.com/ qadeer/cav-issta.htm.

2. Ahmed Bouajjani, Javier Esparza, and Oded Maler. Reachability analysis of pushdown automata: Application to model-checking. In CONCUR, LNCS 1243, pages 135-150, 1997.

3. Ahmed Bouajjani, Javier Esparza, and Tayssir Touili. A generic approach to the static analysis of concurrent programs with procedures. In IJFCS, volume 14(4), pages 551-, 2003.

4. M. B. Dwyer and L. A. Clarke. Data flow analysis for verifying properties of concurrent programs. In ACM SIGSOFT, pages 62-75, 1994.

5. P. Godefroid. Model checking for programming languages using verisoft. In $P O P L$, pages 174-186, 1997.

6. P. Godefroid and P. Wolper. Using partial orders for efficient verification of deadlockfreedom and safety properties. In Formal Methods in Systems Design, pages 149-164, 1993.

7. T. Henzinger, R. Jhala, R. Mazumdar, and S. Qadeer. Thread-modular abstraction refinement. In $C A V$, LNCS 2725, pages 262-274, 2003.

8. F. Ivančić, Z. Yang, M. Ganai, A. Gupta, and P. Ashar. Efficient SAT-based bounded model checking for software verification. In Symposium on Leveraging Applications of Formal Methods, 2004.

9. S. Qadeer, S. K. Rajamani, and J. Rehof. Summarizing procedures in concurrent programs. In POPL, pages 245-255, 2004.

10. G. Ramalingam. Context-sensitive synchronization-sensitive analysis is undecidable. In ACM Trans. Program. Lang. Syst., volume 22(2), pages 416-430, 2000.

11. T. W. Reps, S. Horwitz, and S. Sagiv. Precise interprocedural dataflow analysis via graph reachability. In POPL, pages 49-61, 1985.

12. D. A. Schmidt and B. Steffen. Program analysis as model checking of abstract interpretations. In Static Analysis, 5th International Symposium, LNCS 1503, pages 351-380, 1998.

13. S. D. Stoller. Model-checking multi-threaded distributed java programs. In STTT, volume 4(1), pages 71-91, 2002.

14. W. Visser, K. Havelund, G. P. Brat, S. Park, and F. Lerda. Model checking programs. In Automated Software Engineering, volume 10(2), pages 203-232, 2003.

15. I. Walukeiwicz. Model checking ctl properties of pushdown systems. In FSTTCS, LNCS 1974, pages 127-138, 2000. 


\section{Appendix}

Proposition 1 (Double-Indexed Reduction Result) Given a concurrent program $\mathcal{C P}$ comprised of the $n$ threads $T_{1}, \ldots, T_{n}, \mathcal{C P} \models \mathrm{E}_{\text {fin }} h(i, j)$ iff $\mathcal{C P}(i, j) \models \mathrm{E}_{\text {fin }} h(i, j)$, where $\mathcal{C P}(i, j)$ is the concurrent program comprised solely of the threads $T_{i}$ and $T_{j}$.

Proof Idea We show a stuttering path correspondence between the finite computation paths of $\mathcal{C P}$ and $\mathcal{C P}(i, j)$. Given a finite computation $x$ of $\mathcal{C P}$, we construct a finite computation path $y$ of $\mathcal{C P}(i, j)$ such that $x[i, j]$, viz., $x$ projected on to the local computation paths of $T_{i}$ and $T_{j}$, is stuttering equivalent to $y[i, j]$, and vice versa.

$\Rightarrow$ Given a finite computation $x$ of $\mathcal{C P}$, we construct a finite stuttering computation $y$ of $\mathcal{C}(i, j)$ by letting threads $T_{i}$ and $T_{j}$ execute the same local transitions as were executed along $x$. We need to show that all transitions fired along $y$ are enabled. Towards that end we note that since in $\mathcal{C P}(i, j)$ there are no threads other than $T_{i}$ and $T_{j}$, there is lesser competition for acquisition of locks than in $\mathcal{C P}$. Thus all transitions that were enabled along $x$ are also enabled along $y$.

$(\Leftarrow)$ The construction is similar to the first part. Given a finite computation $y$ of $\mathcal{C P}(i, j)$ we can construct a finite computation $x$ of $\mathcal{C P}$ by letting threads $T_{i}$ and $T_{j}$ execute the same transitions as were executed along $y$ and by letting the rest of the threads stutter in their initial states. Since threads other than $T_{i}$ and $T_{j}$ do not execute any transitions to acquire locks, they do not offer any competition to either $T_{i}$ or $T_{j}$. Thus all transitions of $T_{i}$ and $T_{j}$ that were enabled along $y$ are also enabled along $x$.

Theorem 5 (Decomposition Result) Let $\mathcal{C P}$ be a concurrent program comprised of the two threads $T_{1}$ and $T_{2}$ with nested locks. Then for control states $a_{1}$ and $b_{2}$ of $T_{1}$ and $T_{2}$, respectively, $\mathcal{C P} \models \operatorname{EF}\left(a_{1} \wedge b_{2}\right)$ iff there are computations $x$ and $y$ of the individual threads $T_{1}$ and $T_{2}$, respectively, leading to configurations $s$ with $T_{1}$ in control state $a_{1}$ and $t$ with $T_{2}$ in control state $b_{2}$ such that

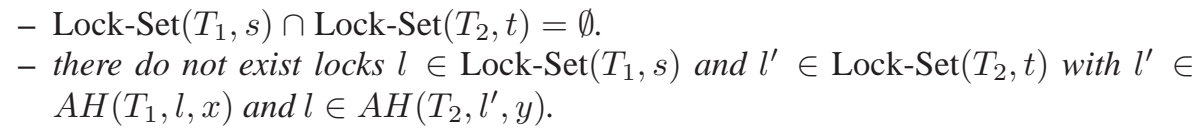

\section{Proof}

$(\Rightarrow)$ Since $\mathcal{C P} \models \operatorname{EF}\left(a_{1} \wedge b_{2}\right)$, there exists a finite path $z$ of $\mathcal{C P}$ leading to a global configuration $u$ with $T_{1}$ and $T_{2}$ in local control states $a_{1}$ and $b_{2}$, respectively. Let $x$ and $y$ be the finite sequences that we get from $z$ by projecting on to the local computations of $T_{1}$ and $T_{2}$, respectively. Projection of a global configuration $\left(\langle a, v\rangle,\langle b, w\rangle, l_{1}, \ldots, l_{m}\right)$ of $\mathcal{C P}$ on to thread $T_{1}$, say, results in the configuration $\left(\langle a, v\rangle, l_{1}^{\prime}, \ldots, l_{m}^{\prime}\right)$, wherein we drop the local configuration of the other thread (in this case $T_{2}$ ) and free all the locks currently held by $T_{2}$. Thus for $1 \leq i \leq m, l_{i}^{\prime}=\perp$ if $l_{i}=2$ and $l_{i}^{\prime}=l_{i}$, otherwise. Simple projection as described above might result in stuttering sequence. This happens when we project $z_{i}$ and $z_{i+1}$ on to $T_{1}$ whereas the transition $z_{i} \rightarrow z_{i+1}$ was fired locally by $T_{2}$ and so it caused no change in the local configuration of $T_{1}$. Thus the projections of both $z_{i}$ and $z_{i+1}$ on to $T_{1}$ are exactly the same. It is easy to see that by removing these stuttering we get valid computations $x$ and $y$ of $T_{1}$ and $T_{2}$, respectively. Let $s$ 
and $t$ be the last configurations occurring along $x$ and $y$, respectively. Then since both threads $T_{1}$ and $T_{2}$ cannot simultaneously hold the same lock, we have $\operatorname{Lock}-\operatorname{Set}\left(T_{1}, s\right)$ $\cap \operatorname{Lock}$-set $\left(T_{2}, t\right)=\emptyset$ thereby proving the first part of the result.

To prove the second part we argue by contradiction. If possible, suppose that there exist locks $l \in \operatorname{Lock-Set}(s, x)$ and $l^{\prime} \in \operatorname{Lock-Set}(t, y)$ such that $l \in A H\left(T_{2}, l^{\prime}, y\right)$ and $l^{\prime} \in A H\left(T_{1}, l, x\right)$. Let $t r: y_{i} \stackrel{\text { acquire }\left(l^{\prime}\right)}{\longrightarrow} y_{i+1}$ be the last transition to acquire $l^{\prime}$ that was fired along $y$. Since $l^{\prime} \in L^{\prime}$, we have that $t r$ is also the last local transition to acquire $l^{\prime}$ that was fired along $z$. Furthermore, since $l \in A H\left(T_{2}, l^{\prime}, y\right)$ there exists a transition of the form $t r^{\prime}: y_{j} \stackrel{\text { acquire }(l)}{\longrightarrow} y_{j+1}$ where $j>i$, that was fired along $y$. Since the relative order of firing of local transitions along $y$ is the same as along $z$, from the above observation we have that the last transition to acquire $l$ along $z$ was fired after $t r$, viz., the last transition to acquire $l^{\prime}$ along $z$. Similarly, using the fact that $l^{\prime} \in A H\left(T_{1}, l, x\right)$, we can deduce that the last transition to acquire $l^{\prime}$ along $z$ is fired after the last transition to acquire $l$ which contradicts the previous observation. This completes the proof of the second part of the result.

$(\Leftarrow)$ Let $x$ be a computation of $T_{1}$ leading to configuration $s$ of the form $(\langle a, v\rangle$, $\left.l_{1}, \ldots, l_{m}\right)$ and $y$ a computation of $T_{2}$ leading to a configuration of the form $\left(\left\langle b_{2}\right.\right.$, $\left.w\rangle, l_{1}^{\prime}, \ldots, l_{m}^{\prime}\right)$. Let $m$ be the maximum value of the lengths of $x$ and $y$. We show by induction on $m$ that there exists a computation $z$ of $\mathcal{C P}$ leading to a configuration $u=\left(\left\langle a_{1}, v\right\rangle,\left\langle b_{2}, w\right\rangle, l_{1}^{\prime \prime}, \ldots, l_{m}^{\prime \prime}\right)$ where for each $k, l_{k}^{\prime \prime}$ equals $i, j$ or $\perp$ accordingly as $l_{k}=i, l_{k}^{\prime}=j$ or $l_{k}=\perp$ and $l_{k}^{\prime}=\perp$, respectively.

The base case where $x$ and $y$ are just comprised of the initial configurations of $T_{1}$ and $T_{2}$ is trivial as the computation comprising of the initial configuration $z=\left(\mathrm{i}_{1}, \mathrm{i}_{2}, \perp\right.$ $, \ldots, \perp)$ of $\mathcal{C P}$, where $i_{1}$ and $i_{2}$ are respectively the initial configurations of threads $T_{1}$ and $T_{2}$, suffices. Let $m>1$ and assume that the result is true for all pairs of paths with each path having length less than $m$. Let $x=x_{1}, \ldots, x_{n}$ and $y=y_{1}, \ldots, y_{n^{\prime}}$, where $m$ is the maximum of $n$ and $n^{\prime}$, and let $L=\operatorname{Lock}-\operatorname{Set}\left(T_{1}, x_{n}\right)$ and $L^{\prime}=\operatorname{Lock}-\operatorname{Set}\left(T_{2}, y_{n^{\prime}}\right)$.

We first consider the scenario where $L=\emptyset$. In this case we can construct the desired computation $z$ by first scheduling all the transitions fired along $x$ resulting in global configuration $u^{\prime}$, say, of $\mathcal{C P}$. Since $L=\emptyset, u^{\prime}=\left(\left\langle a_{1}, v\right\rangle, \mathrm{i}_{2}, \perp, \ldots, \perp\right)$, where $\mathrm{i}_{2}$ is the initial configuration of $T_{2}$. Notice that in $u^{\prime}$ all the locks are free, viz., not in the possession of any thread. Hence starting at $u^{\prime}$, we can schedule all the transitions fired along $y$. All these transitions are enabled as $T_{1}$ now offers no competition to $T_{2}$ by trying to acquire locks. This yields the desired computation $z$ ending in the global configuration $u=\left(\left\langle a_{1}, v\right\rangle,\left\langle b_{2}, w\right\rangle, l_{1}^{\prime \prime}, \ldots, l_{m}^{\prime \prime}\right)$ thus completing the induction step for this case. The case where $L^{\prime}=\emptyset$ is handled similarly.

Now assume that $L$ and $L^{\prime}$ are both non-empty. Let $x^{p}=x_{1}, \ldots, x_{g}$ be the longest prefix of $x$ with the property that $\operatorname{Lock-\operatorname {Set}}\left(T_{1}, x_{g}\right)=\emptyset$. Let $y^{p}=y_{1}, \ldots, y_{h}$ be analogously defined. Then by the induction hypothesis, there exists a finite computation path $z^{\prime}$ of $\mathcal{C P}$ leading to configuration $u^{\prime}$ with $T_{1}$ and $T_{2}$ in the same local configurations as in $x_{g}$ and $y_{h}$, respectively, and with all the locks free. Let transition $x_{i} \rightarrow x_{i+1}$ of $T_{1}$ be denoted by $t r_{i}$ and transition $y_{i} \rightarrow y_{i+1}$ be denoted by $t r_{i}^{\prime}$. Let $l_{1}, \ldots, l_{|L|}$ be an enumeration of the locks in $\operatorname{Lock-\operatorname {Set}}\left(T_{1}, s\right)$ and $l_{1}^{\prime}, \ldots, l_{\left|L^{\prime}\right|}^{\prime}$ an enumeration of the locks in $\operatorname{Lock-Set}\left(T_{2}, t\right)$. Assume that $l_{1}, \ldots, l_{|L|}$ were acquired for the last time along $x_{g}, \ldots, x_{n}$ by transitions $t r_{i n_{1}}, \ldots, t r_{i n_{|L|}}$, respectively, listed in increasing order of in- 
dices. Similarly assume that $l_{1}^{\prime}, \ldots, l_{\left|L^{\prime}\right|}^{\prime}$ were acquired for the last time along $y_{h}, \ldots, y_{n^{\prime}}$ by $i n_{1}^{\prime}, \ldots, i n_{\left|L^{\prime}\right|}^{\prime}$, respectively.

To get the required computation $z$, beginning at $u^{\prime}$, we need to merge the firing of the two sequences $t r_{g}, \ldots, t r_{n-1}$ and $t r_{h}^{\prime}, \ldots, t r_{n^{\prime}-1}^{\prime}$ while preserving the relative ordering of the transitions belonging to the same sequence. Note that once transition $t r_{i n_{j}}$ acquiring lock $l_{j} \in L$ is fired by $T_{1}$ then, by definition of $t r_{i n_{j}}$, thread $T_{1}$ does not release $l_{j}$ thereafter. Thus before we fire $t r_{i n_{j}}$ along $z$ we must ensure that all the transitions fired along $y_{h}, \ldots, y_{n^{\prime}}$ that acquire or release lock $l_{j}$ have already been executed. Let $t r_{\max \left(l_{j}\right)}^{\prime}$ be the last transition fired along $y$ that releases $l_{j}$. Then all transitions $t r_{h}^{\prime}, \ldots, t r_{\max \left(l_{j}\right)}^{\prime}$ must be fired before $t r_{i n_{j}}$ is fired along $z$. In this fashion, we get a fire-before relation among the transitions $t r_{g}, \ldots, t r_{n-1}, t r_{h}^{\prime}, . ., t r_{n^{\prime}-1}^{\prime}$. This relation can be captured as a directed graph with the transitions listed above as nodes and an edge from transition trans to trans' if trans must be fired before trans' along $z$. It is easy to see that there is a scheduling of transitions starting at $u^{\prime}$ that merges $t r_{g}, \ldots, t r_{n-1}$ and $t r_{h}^{\prime}, \ldots, t r_{n^{\prime}-1}^{\prime}$ in a consistent fashion iff this graph is acyclic which is guaranteed by condition 2 in the statement of the theorem. Finally, to construct $z$ we first get a total ordering of the transition $t r_{g}, \ldots, t r_{n-1}, t r_{h}^{\prime}, \ldots, t r_{n^{\prime}-1}^{\prime}$ and schedule the firing of these transitions in this order beginning at $u^{\prime}$. This yields the desired computation and completes the induction step.

Proposition 6 Given a concurrent program $\mathcal{C P}$ comprised of threads $T$ and $T^{\prime}$, the model checking problem for pairwise reachability, viz., formulas of the form $\operatorname{EF}\left(a_{1} \wedge b_{2}\right)$ is decidable.

Proof From the previous result, it suffices to check whether there exist $L, L^{\prime}, A H_{1}$, ..., $A H_{m}, A H_{1}^{\prime}, \ldots, A H_{m}^{\prime} \subseteq\{1, \ldots, m\}$ such that (i) $L \cap L^{\prime}=\emptyset$, and (ii) $T_{A} \models$ $\mathrm{EF}\left(a_{1}, L, A H_{1}, \ldots, A H_{m}\right)$ and $T_{A}^{\prime} \models \operatorname{EF}\left(b_{2}, L^{\prime}, A H_{1}^{\prime}, \ldots, A H_{m}^{\prime}\right)$ and (iii) there do not exist $l_{i} \in L$ and $l_{j} \in L^{\prime}$ such that $j \in A H_{i}$ and $i \in A H_{j}^{\prime}$. Note that we have reduced the problem of pairwise reachability to instances of reachability for the individual pushdown automata $T_{A}$ and $T_{A}^{\prime}$ - one for each valuation of the sets $L, L^{\prime}$, $A H_{1}, \ldots, A H_{m}, A H_{1}^{\prime}, \ldots, A H_{m}^{\prime}$. Since the model checking problem for pushdown systems is known to be decidable and there are only finitely many choices for $L, L^{\prime}$, $A H_{1}, \ldots, A H_{m}, A H_{1}^{\prime}, \ldots, A H_{m}^{\prime}$ the decidability of pairwise reachability follows.

\section{Undecidability for Non-nested Locks}

In this section, we show that for concurrent programs comprised of two threads $T_{1}$ and $T_{2}$ communicating via locks (not necessarily nested), the model checking problem for pairwise reachability, viz., properties of the form $\operatorname{EF}\left(a_{1} \wedge b_{2}\right)$, where $a_{1}$ and $b_{2}$ are control states of $T_{1}$ and $T_{2}$, respectively, is undecidable.

Given a concurrent program $\mathcal{C P}$ comprised of two threads $T_{1}$ and $T_{2}$ communicating via pairwise rendezvous, we construct a new concurrent program $\mathcal{C} \mathcal{P}^{\prime}$ comprised of threads $T_{1}$ and $T_{2}$ such that the set of control states of $T_{1}$ and $T_{2}$ are supersets of the sets of control states of $T_{1}$ and $T_{2}$, respectively, and for control states $a_{1}$ and $b_{2}$ of $T_{1}$ and $T_{2}$, respectively, $\mathcal{C P} \models \operatorname{EF}\left(a_{1} \wedge b_{2}\right)$ iff $\mathcal{C} \mathcal{P}^{\prime} \models \operatorname{EF}\left(a_{1} \wedge b_{2}\right)$. This reduces the 
decision problem for pairwise reachability for threads communicating via pairwise rendezvous to threads communicating via locks. But since pairwise reachability for threads communicating via pairwise rendezvous is undecidable, our result follows.

Simulating Pairwise Rendezvous using Locks We now present the key idea behind the simulation leaving the details for the appendix. We show how to simulate a given pair $a \stackrel{m !}{\longrightarrow} b$ and $c \stackrel{m ?}{\longrightarrow} d$ of send and receive pairwise rendezvous transitions, respectively. Recall that for this rendezvous to be executed, both the send and receive transitions must be simultaneously enabled, else neither transition can fire. We assume, without loss of generality, that each pair of send and receive transitions in the two threads is labeled uniquely and that each send(receive) transition in one thread has a matching receive(send) transition in the other. Then corresponding to the labels $m$ ! and $m$ ?, we introduce the locks $l_{m !}, l_{m}$ ? and $l_{m}$. Given $i \in\{1,2\}$, let $i^{\prime} \in\{1,2\}$ be such that $i \neq i^{\prime}$. Let $i_{i}$ be the initial control state of $T_{i}$. Then in $\mathrm{T}_{i}$ we introduce a new initial state $\mathrm{i}_{i}^{\prime}$. Starting from $\mathrm{i}_{i}^{\prime}$, we first let thread $\mathrm{T}_{i}$ acquire all locks of the form $l_{l a b}$, where $T_{i^{\prime}}$ has a rendezvous send or receive transition labeled with $l a b$. Once the locks corresponding to all these labels have been acquired, $T_{i}$ reaches the state $i_{i}$, i.e., the initial state of thread $T_{i}$. Thus before $\mathrm{T}_{i}$ starts simulating $T_{i}$ it must acquire all locks corresponding to send/receive rendezvous transitions in $T_{i^{\prime}}$ in some pre-determined order. Thus the state transition digram of $\mathrm{T}_{j}$ has a unique path of lock-acquisition transitions from states $i_{j}^{\prime}$ to $i_{j}$. The states and transitions occurring along this path are called preliminary and the remaining non-preliminary.

Consider now the send transition $t r: a \stackrel{m !}{\longrightarrow} b$ of thread $T_{1}$, say. By the above construction, we have that before $\mathrm{T}_{1}$ starts mimicking $t r$ in local state $a$ it already has possession of lock $l_{m}$ ? Then $\mathrm{T}_{1}$ simulates $T_{1}$ via the following sequence of transitions: $a \stackrel{\text { acquire }\left(l_{m}\right)}{\longrightarrow} a_{1} \stackrel{\text { release }\left(l_{m}\right)}{\longrightarrow} a_{2} \stackrel{\text { acquire }\left(l_{m !}\right)}{\longrightarrow} a_{3} \stackrel{\text { release }\left(l_{m}\right)}{\longrightarrow} b \stackrel{\text { acquire }\left(l_{m}\right)}{\longrightarrow}$ $b_{1} \stackrel{\text { release }\left(l_{m !}\right)}{\longrightarrow} b_{2}$. Similarly we assume that $\mathrm{T}_{2}$ has possession of $l_{m}$ ! before it starts mimicking $t r^{\prime}: c \stackrel{m ?}{\longrightarrow} d$. Then $\mathrm{T}_{2}$ simulates $t r^{\prime}$ by firing the following sequence of transitions: $c \stackrel{\text { acquire }\left(l_{m} \text { ? }\right)}{\longrightarrow} c_{1} \stackrel{\text { release }\left(l_{m !}\right)}{\longrightarrow} c_{2} \stackrel{\text { acquire }\left(l_{m}\right)}{\longrightarrow} c_{3} \stackrel{\text { release }\left(l_{m ?}\right)}{\longrightarrow} d \stackrel{\text { acquire }\left(l_{m !}\right)}{\longrightarrow}$ $d_{1} \stackrel{\text { release }\left(l_{m}\right)}{\longrightarrow} d_{2}$.

The reason for letting thread $\mathrm{T}_{1}$ acquire $l_{m}$ ? at the outset is to prevent thread $\mathrm{T}_{2}$ from firing transition $c \stackrel{m ?}{\longrightarrow} d$ without synchronizing with $t r: a \stackrel{m !}{\longrightarrow} d$. To initiate execution of the pairwise rendezvous involving $t r$, thread $\mathrm{T}_{1}$ releases lock $l_{m}$ ? and only when lock $l_{m}$ ? is released can $\mathrm{T}_{2}$ pick it up in order to execute the matching receive transition labeled with $m$ ?. But before $\mathrm{T}_{1}$ releases $l_{m}$ ? it acquires $l_{m}$. Note that this trick involving chaining wherein before releasing a lock a thread is forced to pick up another lock gives us the ability to introduce a relative ordering on the firing of local transitions of $T_{1}$ and $T_{2}$ which in turn allows us to simulate (in a weak sense) the firing of the pairwise rendezvous comprised of $t r$ and $t r^{\prime}$. It can be seen that due to chaining, the local transitions in the two sequences defined above can only be fired in the following order: $a \stackrel{\text { acquire }\left(l_{m}\right)}{\longrightarrow} a_{1}, a_{1} \stackrel{\text { release }\left(l_{m} \text { ? }\right)}{\longrightarrow} a_{2}, \stackrel{\text { acquire }\left(l_{m} \text { ? }\right)}{\longrightarrow} c_{1}, c_{1} \stackrel{\text { release }\left(l_{m !}\right)}{\longrightarrow} c_{2}$, 
$a_{2} \stackrel{\text { acquire }\left(l_{m !}\right)}{\longrightarrow} a_{3} a_{3} \stackrel{\text { release }\left(l_{m}\right)}{\longrightarrow} b, c_{2} \stackrel{\text { acquire }\left(l_{m}\right)}{\longrightarrow} c_{3}, c_{3} \stackrel{\text { release }\left(l_{m}\right)}{\longrightarrow} d, b \stackrel{\text { acquire }\left(l_{m}\right)}{\longrightarrow} b_{1}$,
$b_{1} \stackrel{\text { release }\left(l_{m !}\right)}{\longrightarrow} b_{2}, d \stackrel{\text { acquire }\left(l_{m !}\right)}{\longrightarrow} d_{1}, d_{1} \stackrel{\text { release }\left(l_{m}\right)}{\longrightarrow} d_{2}$.

It is important to note that the use of overlapping locks is essential in implementing chaining thereby forcing a pre-determined order of firing of the local transitions which cannot be accomplished using nested locks alone. Although the above construction captures the key idea behind the simulation, there are other technical intricacies that require augmentation of the above construction and are discussed in the appendix.

Our construction is complete except for the minor caveat that it works only if we can ensure program $\mathcal{C} \mathcal{P}^{\prime}$ reaches a configuration wherein threads $T_{1}$ and $T_{2}$ are both in non-preliminary control states having executed all the preliminary transitions and acquired the initial locks else the simulation will not be faithful. This is because we can let thread $\mathrm{T}_{1}$ execute any sequence of transitions (even the mimicked rendezvous transitions) without being blocked by letting $T_{2}$ remain in its initial state $i_{2}^{\prime}$ and thereby not acquire any locks at all thus providing no competition for locks to thread $\mathrm{T}_{1}$. Thus $\mathcal{C} \mathcal{P}^{\prime}$ exhibits more behaviors than $\mathcal{C P}$. So while $\mathcal{C P} \models \operatorname{EF}\left(a_{1} \wedge b_{2}\right)$ implies $\mathcal{C} \mathcal{P}^{\prime} \models$ $\mathrm{EF}\left(a_{1} \wedge b_{2}\right)$, the other direction need not be true.

To fix the above problem, we now introduce two new lock $l_{1}$ and $l_{2}$ and then modify the state transition graphs of $T_{1}$ and $T_{2}$ as follows: We first let $T_{1}$ acquire $l_{1}$ in state $\mathrm{i}_{1}^{\prime}$ before it starts executing all the preliminary lock acquisition transitions as described above. After all these locks have been acquired we let it release $l_{1}$ and then acquire $l_{2}$ to end up in state $i_{1}$. Furthermore, we introduce a new control state designated as terminal $_{1}$ from which there is no out-going transition and from each local state $c$ of $\mathrm{T}_{1}$ reachable from $\mathrm{i}_{1}$ we introduce a transition of the form $c \stackrel{\text { release }\left(l_{2}\right)}{\longrightarrow}$ terminal $_{1}$. The state transition diagram of $T_{2}$ is modified analogously. The modifications are shown in figure 1.

Note that once $T_{2}$ acquires lock $l_{1}$, to relinquish it, $T_{2}$ must fire a transition leading to the state terminal $_{1}$. This forces $T_{1}$ to fire all the preliminary lock-acquisition transitions before $T_{2}$ can fire the transition to acquire $l_{1}$ for otherwise $T_{1}$ can enter a non-preliminary state only when $\mathrm{T}_{2}$ enters the terminal state and releases lock $l_{1}$. Thus to ensure $\mathcal{C} \mathcal{P}^{\prime} \models \operatorname{EF}\left(a_{1} \wedge b_{2}\right)$, where $a_{1}$ and $b_{2}$ are control states of $T_{1}$ and $T_{2}$, respectively, and thus non-terminal and non-initial states of $T_{1}$ and $T_{2}$, we must ensure that $T_{1}$ must fire all the preliminary lock-acquisition transitions before $T_{2}$ can fire the transition to acquire $l_{1}$. This fixes the problem described before and gives us the following result. Since the model checking problem for pairwise reachability is known to be undecidable for thread communicating using pairwise rendezvous and since we can, by the above result, simulate pairwise rendezvous using locks in a way so as to preserve pairwise reachability, we have the following undecidability result.

Theorem 8 The model checking problem for pairwise reachability is undecidable for concurrent programs comprised of two threads communicating using locks. 
Thread T1

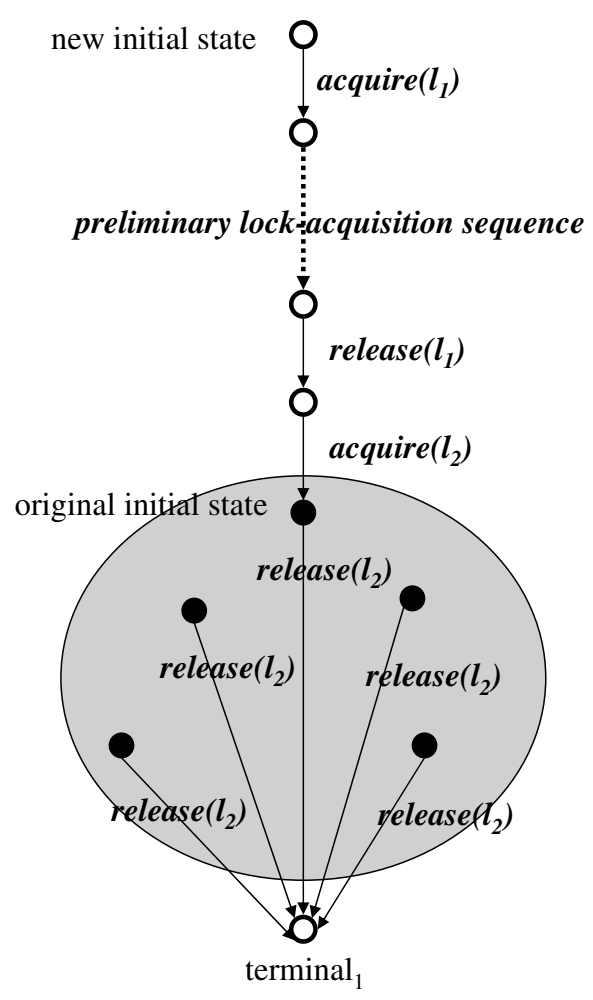

Thread T2

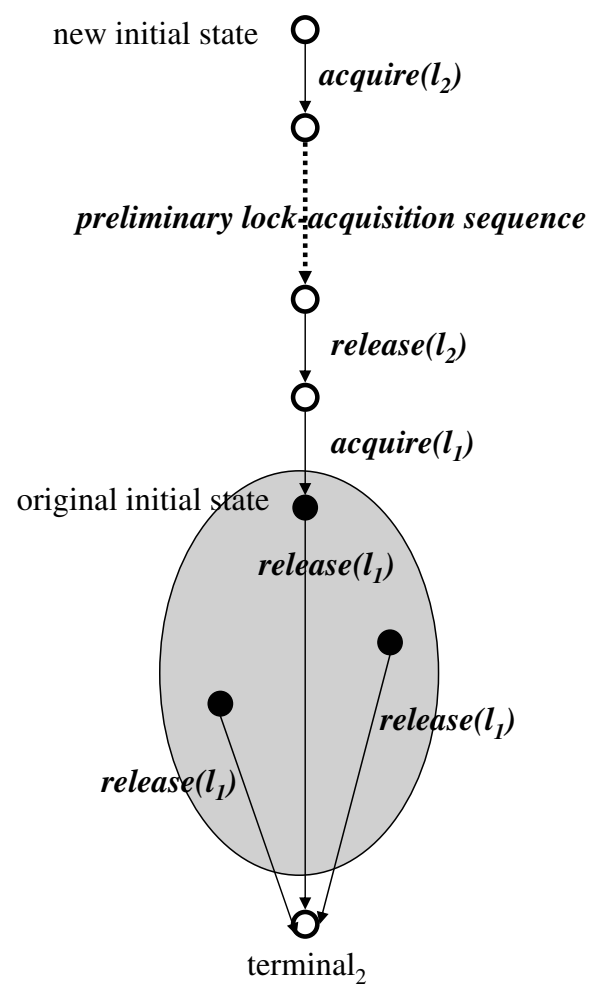

Fig. 1. The Transition diagram for augmented threads 